\title{
Genes related to inflammation and bone loss process in periodontitis suggested by bioinformatics methods
}

\author{
Liang Song ${ }^{1 *}$, Jueqi Yao ${ }^{2}$, Zhijing $\mathrm{He}^{3}$ and Bin $\mathrm{Xu}^{1}$
}

\begin{abstract}
Background: Despite of numerous studies on periodontitis, the mechanism underlying the progression of periodontitis still remains largely unknown. This study aimed to have an expression profiling comparison between periodontitis and normal control and to identify more candidate genes involved in periodontitis and to gain more insights into the molecular mechanisms of periodontitis progression.

Methods: The gene expression profile of GSE16134, comprising 241 gingival tissue specimens and 69 healthy samples as control which were obtained from 120 systemically healthy patients with periodontitis (65 with chronic and 55 with aggressive periodontitis), was downloaded from the Gene Expression Omnibus (GEO) database. Differentially expressed genes (DEGs) in periodontitis samples were screened using the limma package in R compared with control samples. Gene Ontology (GO) and pathway enrichment analysis upon the DEGs were carried out using Hypergeometric Distribution test. Protein-protein interaction (PPI) network of the DEGs was constructed using Cytoscape, followed by module selection from the PPI network using MCODE plugin. Moreover, transcription factors (TFs) of these DEGs were identified based on TRANSFAC database and then a regulatory network was constructed.
\end{abstract}

Results: Totally, 762 DEGs (507 up- and 255 down-regulated) in periodontitis samples were identified. DEGs were enriched in different GO terms and pathways, such as immune system process, cell activation biological processes, cytokine-cytokine receptor interaction, and metabolic pathways. Cathepsin S (CTSS) and pleckstrin (PLEK) were the hub proteins in the PPI network and 3 significant modules were selected. Moreover, 19 TFs were identified including interferon regulatory factor 8 (IRF8), and FBJ murine osteosarcoma viral oncogene homolog B (FOSB).

Conclusion: This study identified genes (CTSS, PLEK, IRF-8, PTGS2, and FOSB) that may be involved in the development and progression of periodontitis.

\section{Background}

Periodontitis is a chronic inflammatory disease involving interactions between complex microbial biofilms, many cell populations and inflammatory mediators, leading to the destruction of the tooth-supporting structures like the periodontal ligament and the alveolar bone [1]. Besides being a common cause of tooth loss, severe periodontitis (about $8.5 \%$ of patients) can detrimentally affect systemic health, as it can increase the patients' risk for diabetes, atherosclerosis, rheumatoid arthritis, and adverse pregnancy outcomes [2-4]. Two major clinical

\footnotetext{
* Correspondence: slenll@163.com

'Department of Stomatology, The Fifth People's Hospital of Shanghai, Fudan University, No.128, Ruili Rd, Minhang District, Shanghai 200240, China Full list of author information is available at the end of the article
}

entities of periodontitis are currently recognized: chronic periodontitis, which is more common, and aggressive periodontitis, a clinically challenging entity featured by an early onset and a rapid progression [5]. The underlying etiology of both the two forms has not been fully elucidated. Therefore, gaining further insights into the molecular mechanisms of periodontitis will be of great significance for the treatment of periodontitis.

Previous studies have demonstrated that factors that may determine the presence and rate of progression of periodontitis are complex, which can be defined as the interplay of numerous parameters acting simultaneously and unpredictably [1]. For instance, the tooth-associated microbial biofilm or dental plaque is essential but not sufficient to induce periodontitis. The host inflammatory 
response to the microbial challenge can finally cause the destruction of the periodontium [6]. Inflammation and bone loss are hallmarks of periodontal disease [7] and accumulated evidence demonstrates that a number of mediators are involved in these processes [8]. Cochran et al. had reported that the reduction of inflammation and attenuation of the host's immune reaction to the microbial plaque could lead to a decrease in the ratio of nuclear factor-kappa B ligand (RANKL)/osteoprotegerin (OPG) and a decrease in associated bone loss [7]. Besides, a study has reviewed that cytokines such as interleukin-1 (IL-1) and tumor necrosis factor (TNF) are a significant and integral component of the host reaction to periodontal infection [8]. In addition, secreted IL-8 induced by multiple stimuli like live bacteria and proinflammatory cytokines is associated with the inflammation and invasiveness of periodontitis [9]. Despite of numerous investigations on periodontitis, the mechanism still remains largely unknown.

Using the same gene expression profile, Stoecklin et al. identified specific miRNAs (has-miR-210 and hsamiR-185) and their target genes in gingival tissues [10]. In addition, Kebschull et al. found that small differences in gene expression and the highly variable classifier performance suggested limited dissimilarities between established chronic periodontitis and aggressive periodontitis lesions [11]. We sought to have an expression profiling comparison between periodontitis (chronic periodontitis and aggressive periodontitis collectively) and normal control, identifying more candidate genes involved in both chronic and aggressive periodontitis and to gain more insights into the molecular mechanisms of periodontitis progression.

\section{Methods}

\section{Microarray data and data preprocessing}

The gene expression profile data of GSE16134 [11] was downloaded from the Gene Expression Omnibus (GEO) in NCBI (http://www.ncbi.nlm.nih.gov/geo/) based on the platform of GPL570 [HG-U133_Plus_2] Affymetrix Human Genome U133 Plus 2.0 Array. This dataset included 241 'diseased' gingival tissue specimens (bleeding on probing, probing depth $\geq 4 \mathrm{~mm}$, and clinical attachment loss $\geq 3 \mathrm{~mm}$ ) and 69 'healthy' gingival tissue samples (no bleeding on probing, probing depth $\leq 4 \mathrm{~mm}$, and clinical attachment loss $\leq 4 \mathrm{~mm}$ ), obtained from 120 systemically healthy, non-smoking individuals with moderate/severe periodontitis (65 with chronic and 55 with aggressive periodontitis), as previously described [11, 12]. The 120 patients undergoing periodontal surgery contributed with a minimum of two interproximal gingival papillae from a maxillary posterior and when available, a 'healthy' papilla was obtained. In the present study, samples from chronic and aggressive periodontitis patients were taken together as one group, designed as periodontitis samples, and were compared with the 69 'healthy' gingival tissue samples as controls in the following analysis.

The downloaded profile had been preprocessed which was carried out with background correction, $\log _{2}$ transformation, and quantile normalization using the RMA (Robust Multi-array Analysis) method of affy package in $\mathrm{R}$ [13]. In this study, those probes hybridizing to the same gene were normalized using the preprocessCore package [14]. The gene expression matrix of specimens was received and was used to the follow-up analysis.

\section{Screening of DEGs}

The DEGs in periodontitis samples were screened compared with control samples using the Linear Models for Microarray data (limma) package in R [15]. False discovery rate (FDR) [16] was applied for multiple testing correction using Benjamini and Hochberg method [17]. Threshold for the DEGs were set as FDR $<0.05$ and $\mid \log _{2}$ FC (fold change) $\mid \geq 0.58$.

\section{Function and pathway enrichment analysis of DEGs}

In order to investigate the periodontitis progression on the perspective of functional level, Gene Ontology (GO) and pathway enrichment analysis of the identified DEGs were performed in this study. GO categories such as biological process (BP), molecular function (MF), and cellular component $(\mathrm{CC})$ upon the identified DEGs were enriched from GO databases using the Hypergeometric Distribution test [18]. Also, the pathways that the DEGs involved in were enriched using the Hypergeometric Distribution test from the KEGG (Kyoto Encyclopedia of Genes and Genomes) database [19], which had not been used by Kebschull et al. [12]. The p-value $<0.05$ was chosen as the threshold.

\section{PPI network construction of DEGs and modules selection}

The STRING (Search Tool for the Retrieval of Interacting Genes/Proteins) database, which is a database of known and predicted protein interactions [20], was used to select the interactions among the selected DEGs in this study, which were not used in the study of Kebschull et al. [12]. The PPI network was constructed by functional links between proteins that are experimentally derived, as well as links predicted by co-expression analysis and text mining, or PPIs which had related records in the database. Genes included in the PPI network were all DEGs. Besides, the combined score $\geq 0.4$ were chosen for the PPI network construction. Cytoscape software [21] was used to visualize the constructed network while MCODE [22] was used to select significant modules from the PPI network (Parameters: Degree cutoff: 2, Node score cutoff: 0.2, K-core: 2, 
Max. depth: 100). Furthermore, topological analysis of the PPI network was performed and node degrees of these DEGs were analyzed.

\section{Function annotation of the DEGs and construction of regulatory network}

To identify the DEGs which had the transcriptional regulatory functions, the identified DEGs in this study were analyzed using the TRANSFAC database (http:// www.gene-regulation.com/pub/databases.html) [23], a database comprising data of transcription factors (TFs), their target genes and regulatory binding sites. Moreover, a regulatory network based on the identified TFs and their target DEGs was constructed. While in the study of Kebschull et al. [12], TRANSFAC database had not been used.

\section{Results}

\section{Data preprocessing and DEGs screening}

In the original analysis of Kebschull et al. [12], a total of 248 differentially regulated probes were identified at an absolute fold change of $\geq 1.19$, and 30 overexpressed only one under-expressed probe by an absolute change of $>1.5$ fold were identified in aggressive periodontitis lesions compared with chronic periodontitis lesions. However, in this study, after data preprocessing, 20,303 genes were mapped to the probes. Compared with the control samples, a total of 762 DEGs were identified in the periodontitis samples (Additional file 1: Table S1), including 507 up-regulated genes and 255 down-regulated genes.

\section{GO and pathway enrichment analysis of DEGs}

Functional and pathway enrichment analysis indicated that up-regulated DEGs and down-regulated DEGs in the periodontitis samples were significantly enriched in different GO terms and KEGG pathways (Tables 1 and 2). Top $5 \mathrm{GO}$ terms of up- and down-regulated genes were shown in Table 1, respectively. The up-regulated genes were involved in different GO terms such as cell activation, activation of immune response, chemokine activity, and antigen binding (Table 1A). While the down-regulated genes were associated with the GO terms like skin development, epidermal cell differentiation, intermediate filament, and structural molecule activity (Table 1B). On the other hand, top 10 pathways of up- and down-regulated genes were shown in Table 2, respectively. The pathway enrichment analysis showed that the up-regulated genes were mainly involved in staphylococcus aureus infection and cytokine-cytokine receptor interaction (Table 2A), while the down-regulate genes were mainly associated with the pathways such as metabolic pathways, phagosome, and melanogenesis pathways (Table 2B).
Table 1 The functional analysis of the DEGs

\begin{tabular}{|c|c|c|c|c|}
\hline Category & GO ID & Name & Count & $p$-value \\
\hline \multicolumn{5}{|c|}{ A: the top $5 \mathrm{GO}$ terms of the up-regulated DEGs } \\
\hline BP & GO:0001775 & cell activation & 68 & $<1.00 \mathrm{E}-16$ \\
\hline BP & GO:0002253 & $\begin{array}{l}\text { activation of immune } \\
\text { response }\end{array}$ & 48 & $<1.00 \mathrm{E}-16$ \\
\hline BP & GO:0002376 & immune system process & 165 & $<1.00 \mathrm{E}-16$ \\
\hline $\mathrm{BP}$ & GO:0002682 & $\begin{array}{l}\text { regulation of immune } \\
\text { system process }\end{array}$ & 94 & $<1.00 \mathrm{E}-16$ \\
\hline BP & GO:0002684 & $\begin{array}{l}\text { positive regulation of } \\
\text { immune system process }\end{array}$ & 73 & $<1.00 \mathrm{E}-16$ \\
\hline CC & GO:0005576 & extracellular region & 138 & $<1.00 \mathrm{E}-16$ \\
\hline CC & GO:0005615 & extracellular space & 70 & $<1.00 \mathrm{E}-16$ \\
\hline CC & GO:0044421 & extracellular region part & 86 & $<1.00 \mathrm{E}-16$ \\
\hline CC & GO:0071944 & cell periphery & 191 & $2.64 \mathrm{E}-14$ \\
\hline CC & GO:0005886 & plasma membrane & 186 & $1.49 \mathrm{E}-13$ \\
\hline MF & GO:0008009 & chemokine activity & 10 & 2.35E-07 \\
\hline MF & GO:0003823 & antigen binding & 12 & 4.64E-07 \\
\hline MF & GO:0032403 & protein complex binding & 26 & $4.85 \mathrm{E}-07$ \\
\hline MF & GO:0042379 & $\begin{array}{l}\text { chemokine receptor } \\
\text { binding }\end{array}$ & 10 & $1.11 \mathrm{E}-06$ \\
\hline MF & GO:0005178 & integrin binding & 12 & $1.41 \mathrm{E}-06$ \\
\hline
\end{tabular}

B: the top $5 \mathrm{GO}$ terms of the down-regulated DEGs

BP GO:0043588 skin development 311

BP GO:0008544 epidermis development 280

2.33E-14

$\mathrm{BP}$

GO:0030216 keratinocyte

differentiation

$\mathrm{BP}$

GO:0009913 epidermal cell differentiation

GO:0009888 tissue development

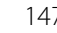

CC GO:0030057 desmosome

CC GO:0005882 intermediate filament

CC GO:0045111 intermediate filament cytoskeleton$$
\mathrm{CC}
$$$$
M F
$$$$
M F
$$$$
\text { NF }
$$$$
M
$$

\begin{tabular}{c|lll} 
MF G0:0016702 & $\begin{array}{l}\text { oxidoreductase activity, } \\
\text { acting on single donors } \\
\text { with incorporation of } \\
\text { molecular oxygen, } \\
\text { incorporation of two }\end{array}$ & & \\
& atoms of oxygen & & \\
MF G0:0016701 & $\begin{array}{l}\text { oxidoreductase activity, 26 } \\
\text { acting on single donors } \\
\text { with incorporation of } \\
\text { molecular oxygen }\end{array}$ & & \\
\hline
\end{tabular}

MF molecular function, BP biological process, $C C$ cell component 
Table 2 The pathway enrichment analysis of the DEGs

\begin{tabular}{|c|c|c|c|}
\hline KEGG ID & Name & Count & $p$-value \\
\hline \multicolumn{4}{|c|}{ A: the top 10 enriched pathways of the up-regulated DEGs } \\
\hline 5150 & Staphylococcus aureus infection & 55 & 4.40E-12 \\
\hline 5144 & Malaria & 51 & $2.12 \mathrm{E}-09$ \\
\hline 4060 & $\begin{array}{l}\text { Cytokine-cytokine } \\
\text { receptor interaction }\end{array}$ & 265 & 2.39E-08 \\
\hline 4141 & $\begin{array}{l}\text { Protein processing in } \\
\text { endoplasmic reticulum }\end{array}$ & 165 & $5.11 \mathrm{E}-07$ \\
\hline 4640 & Hematopoietic cell lineage & 88 & 5.53E-07 \\
\hline 5323 & Rheumatoid arthritis & 91 & $8.66 \mathrm{E}-07$ \\
\hline 5140 & Leishmaniasis & 72 & $1.64 \mathrm{E}-06$ \\
\hline 4670 & $\begin{array}{l}\text { Leukocyte transendothelial } \\
\text { migration }\end{array}$ & 116 & 4.30E-06 \\
\hline 4514 & Cell adhesion molecules (CAMs) & 133 & $6.18 \mathrm{E}-06$ \\
\hline 4062 & Chemokine signaling pathway & 189 & $1.71 \mathrm{E}-05$ \\
\hline \multicolumn{4}{|c|}{ B: the top 10 enriched pathways of the down-regulated DEGs } \\
\hline 590 & Arachidonic acid metabolism & 59 & 0.002776062 \\
\hline 980 & $\begin{array}{l}\text { Metabolism of xenobiotics } \\
\text { by cytochrome P450 }\end{array}$ & 71 & 0.005420335 \\
\hline 982 & $\begin{array}{l}\text { Drug metabolism - } \\
\text { cytochrome P450 }\end{array}$ & 73 & 0.005982341 \\
\hline 350 & Tyrosine metabolism & 41 & 0.007842248 \\
\hline 1100 & Metabolic pathways & 1130 & 0.012102847 \\
\hline 5144 & Malaria & 51 & 0.014274311 \\
\hline 5014 & $\begin{array}{l}\text { Amyotrophic lateral } \\
\text { sclerosis (ALS) }\end{array}$ & 53 & 0.015832167 \\
\hline 4145 & Phagosome & 153 & 0.018215247 \\
\hline 4916 & Melanogenesis & 101 & 0.018240082 \\
\hline 563 & $\begin{array}{l}\text { Glycosylphosphatidylinositol } \\
\text { (GPI)-anchor biosynthesis }\end{array}$ & 25 & 0.025692313 \\
\hline 830 & Retinol metabolism & 64 & 0.026060907 \\
\hline 591 & Linoleic acid metabolism & 30 & 0.036083866 \\
\hline
\end{tabular}

\section{PPI network construction and modules selection}

The PPI network upon the DEGs was shown in Fig. 1. The results of topological analysis showed that interleukin6 (IL-6), cathepsin S (CTSS), and pleckstrin (PLEK) were the hub proteins in the PPI network which had higher node degrees (Fig. 2a). In addition, 3 significant modules were selected from the PPI network (Fig. 2). From the results, we found that most of the genes enriched in the 3 modules were up-regulated. In particular, the top 5 genes with higher node degrees in module 1 were CTSS (degree $=20$ ), chemokine $(\mathrm{C}-\mathrm{C}$ motif) ligand 5 $(C C L 5)($ degree $=19)$, PLEK $($ degree $=19)$, chemokine (C-C motif) receptor 1 (CCR1) (degree $=19)$, and formyl peptide receptor 1 (FPR1) (degree=19) (Fig. 2b, Table 3). The top 5 genes with higher node degrees in module 2 were IL6 (degree = 17), lymphocyte-specific protein tyrosine kinase $(L C K)($ degree $=14)$, Fc fragment of IgE, high affinity I, receptor for; gamma polypeptide $(F C E R 1 G)($ degree $=13), C D 19($ degree $=13)$, and colony stimulating factor 1 receptor $(C S F 1 R)($ degree $=13)$ (Fig. 2c, Table 3). Additionally, the top 5 genes with higher node degrees in module 3 were IL8 (degree $=13$ ), IL1B $($ degree $=12), M M P 9($ degree $=11)$, prostaglandinendoperoxide synthase 2 (PTGS2) (degree=11), and plasminogen activator, tissue (PLAT) (degree=10) (Fig. $2 \mathrm{~d}$, Table 3).

\section{Regulatory network construction}

A total of 9 TFs were identified from the up-regulated DEGs, such as interferon regulatory factor 4 (IRF4), IRF8, and FBJ murine osteosarcoma viral oncogene homolog B (FOSB). Besides, 10 TFs from the downregulated DEGs were selected, such as Kruppel-like factor 4 (KLF4), v-maf avian musculoaponeurotic fibrosarcoma oncogene homolog (MAF), and Meis homeobox 1 (MEIS1). The regulatory network of these TFs and their target genes was shown in Fig. 3. From the results, we found that several DEGs with higher degrees in module 1 could be regulated by IRF8, for example, CTSS, CCL5, and PLEK.

\section{Discussion}

In this study, we used the microarray data to select genes associated with periodontitis. Totally, 762 DEGs in the periodontitis samples were identified compared with the control samples. The up-regulated genes were mainly enriched in the GO terms like cell activation and activation of immune response, as well as the pathways such as staphylococcus aureus infection and cytokinecytokine receptor interaction. The down-regulated genes were mainly linked to tissue development and metabolism pathways. CTSS, PLEK, LCK, and PTGS2 were identified to be hub proteins in the PPI network or in the selected module. Besides, 9 TFs and 10 TFs were selected from the up-regulated genes and down-regulated genes respectively, for example, IRF4, IRF8, and FOSB.

Our results showed that 20,303 genes were mapped to the probes. Compared with the healthy samples, a total of 762 DEGs were identified in the periodontitis samples, including 507 up-regulated genes $(F D R<0.05$ and $\log _{2} \mathrm{FC} \geq 0.58$ ) and 255 down-regulated genes $\left(\mathrm{FDR}<0.05\right.$ and $\left.\log _{2} \mathrm{FC}<-0.58\right)$. While, Kebschull et al. identified a total of 248 differentially regulated probes at an absolute fold change of $\geq 1.19$ [12]. They reported 30 overexpressed and only one under-expressed probe by an absolute change of $>1.5$ fold in aggressive periodontitis lesions compared with chronic periodontitis lesions. Besides, they found that 9258 probes were differentially expressed when compared the 'diseased' tissues with 'healthy' gingival tissues. Collectively, the results showed that we identified distinct genetic 


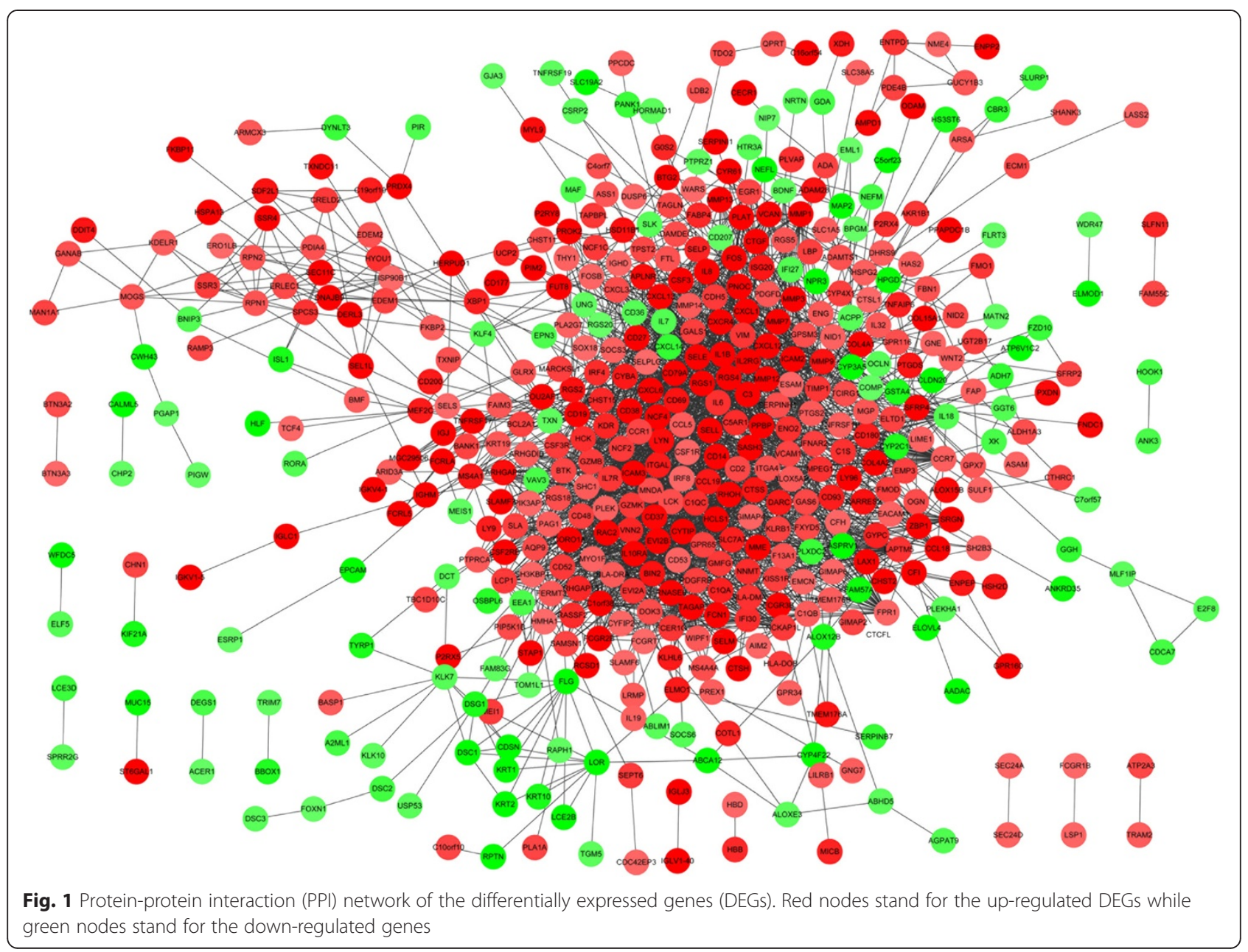

features in periodontitis samples using different screening methods with different thresholds.

In this study, we found that DEGs in periodontitis samples were mainly enriched in different GO terms and pathways, such as cell activation, activation of immune response, staphylococcus aureus infection and cytokinecytokine receptor interaction, using KEGG database which were not used by Kebschull et al. [12]. In their investigations, gene set enrichment analysis was performed and gene sets linked to apoptosis, immune response were enriched in aggressive periodontitis lesions, while genes sets linked to cellular metabolism and epithelial integrity were enriched in chronic periodontitis lesions [12]. In a susceptible host, persistence of bacteria pathogens such as Porphyromonas gingivalis results in aberrant and extended inflammation and subsequent destruction of the tooth-supporting structures [24]. The immune cells such as antigen presenting cells (APC) initially responding to the challenge by bacteria pathogens, including Porphyromonas gingivalis, poised strategically along portals of entry [25]. After recognition of pathogen associated molecular patterns (PAMPs) via pattern recognition receptors (eg, toll like receptors [TLRs]), innate immune cells start responses aiming to clear the inciting agent [26]. Moutsopoulos et al. had showed that Porphyromonas gingivalis could promote $\mathrm{T}$ helper cell 17 (Th17) inducing pathways in chronic periodontitis [24]. Thus, the enrichment results identified in our study was in accordance with the previous studies.

CTSS is a lysosomal cysteine proteinase that may participate in the degradation of antigenic proteins to peptides for presentation on MHC class II molecules [27]. Deficiency of CTSS induces a high bone turnover and then leading to the less dense bone [28]. Mogi et al. demonstrated that the expression level of the key bone degradation enzyme cathepsin $\mathrm{K}$ (another member of family proteins) in gingival crevicular fluid tissues of periodontitis patients was higher than that in normal tissues [29]. Besides, IRF8 can specifically bind to the upstream regulatory region of type I interferon (IFN). Zhao et al. had demonstrated that IRF-8 was a regulator for osteoclastogenesis in bone metabolism [30]. Soft tissue destruction and bone degradation were often found in periodontitis [31]. Moreover, a study revealed that CTSS 


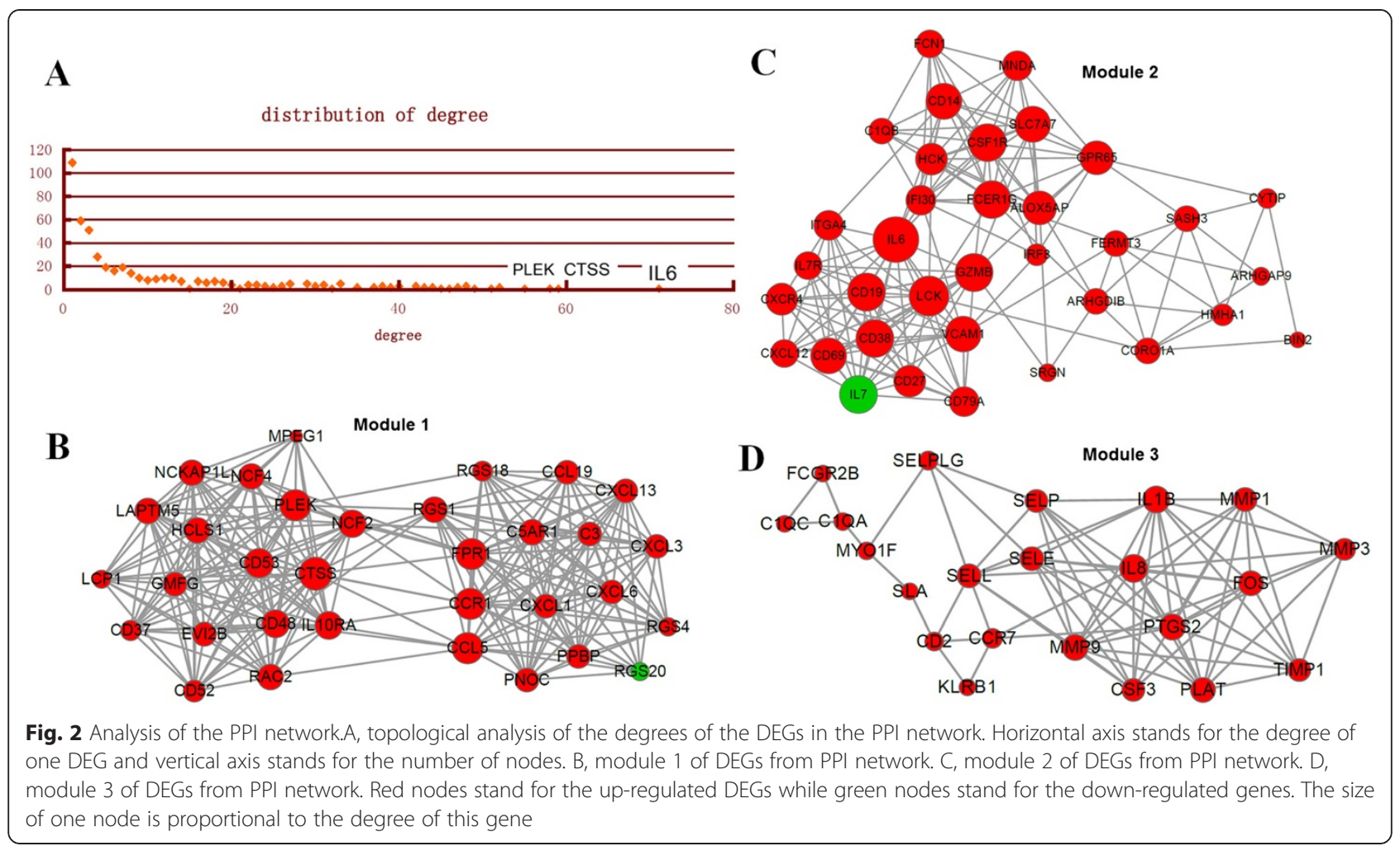

had the binding site for transcription factor IRF1, and combination of IRF8 and IRF1 could promote the CTSS expression [32]. In the present study, CTSS was a hub protein in the PPI network and could be regulate by IRF8 in the regulatory network. In the context, we suggested that CTSS might play an essential role in bone

Table 3 The top 5 DEGs with higher degrees in the selected modules

\begin{tabular}{|c|c|c|c|}
\hline Module & Gene & Expression changes & Degree \\
\hline \multirow[t]{5}{*}{ module 1} & CTSS & up & 20 \\
\hline & CCL5 & up & 19 \\
\hline & PLEK & up & 19 \\
\hline & CCR1 & up & 19 \\
\hline & FPR1 & up & 19 \\
\hline \multirow[t]{5}{*}{ Module 2} & IL6 & up & 17 \\
\hline & LCK & up & 14 \\
\hline & FCER1G & up & 13 \\
\hline & CD19 & up & 13 \\
\hline & CSF1R & up & 13 \\
\hline \multirow[t]{5}{*}{ Module 3} & IL8 & up & 13 \\
\hline & IL1B & up & 12 \\
\hline & MMP9 & up & 11 \\
\hline & PTGS2 & up & 11 \\
\hline & PLAT & up & 10 \\
\hline
\end{tabular}

loss involved in periodontitis progression by interacting with IRF8.

On the other hand, PLEK is a major substrate of protein kinase $\mathrm{C}$ in platelets and leukocytes and appears to play an important role in exocytosis through a currently unknown mechanism [33]. Ding et al. proved that the phosphorylated PLEK increased the secretion of proinflammatory cytokine in mononuclear phagocytes [34]. Besides, Ueki et al. had showed that the secreted monocytes activated by bacterial in gingival crevicular fluid was associated with periodontitis [35]. On the other hand, IRF8 can specifically bind to the upstream regulatory region of IFN [36]. Additionally, Bar-Or et al. showed that B cells could exhibit abnormal proinflammatory cytokine responses (such as exaggerated production of TNF) when activated in the context of the Th1 cytokine IFN [37]. In this study, the results showed that PLEK was a hub protein in the PPI network and could be regulated by IRF8 in the regulatory network. Therefore, we speculated that PLEK might contribute to the periodontitis progression via interacting with $I R F-8$.

PTGS2 is an isozyme of PTGS which is the key enzyme in prostaglandin biosynthesis, and acts both as a dioxygenase and as a peroxidase [38]. The study of Zhang et al. had demonstrated that there was a hypermethylation pattern of the promoter in connection with a lower level of PTGS2 transcription in the inflamed tissues in chronic periodontitis [39]. On the other hand, 


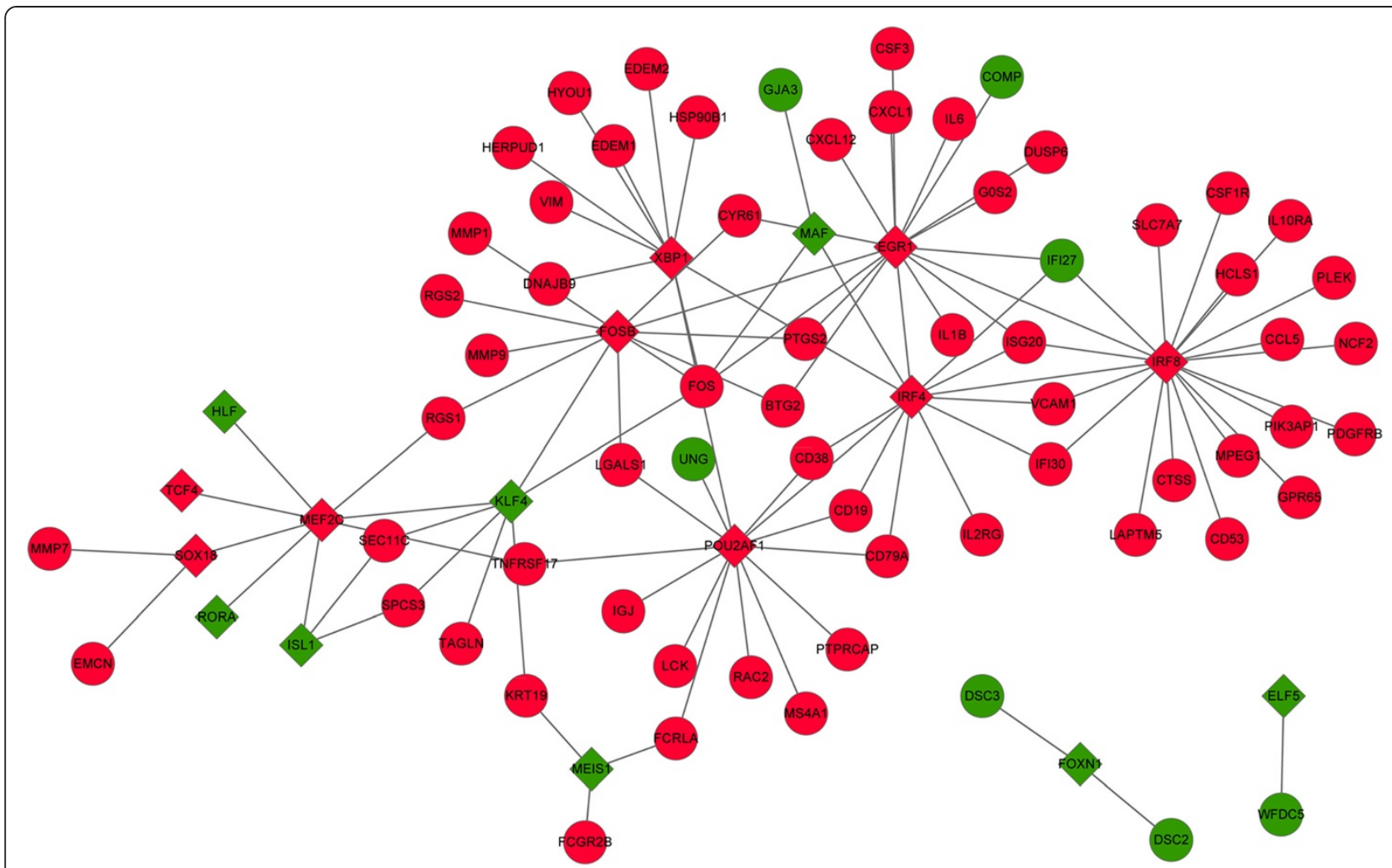

Fig. 3 Regulatory network of the transcription factors-DEGs. Diamond represents the transcription factor while the circle represents the DEG. Red color stands for up-regulated expression while green color stands for down-regulated expression

FOSB is one member of the Fos gene family which encodes leucine zipper proteins that can dimerize with proteins of the JUN family [40]. T cell receptor (TCR)driven early gene expression is controlled by numerous key transcription factors such as FOSB [41]. Additionally, Sreeramkumar et al. had reported that PTGS2 was transcriptionally up-regulated in T cells during TCR/ CD3 triggering and that it behaved as an early inducible gene in the $\mathrm{T}$ cell activation process [42]. Moreover, Chen et al. had demonstrated that costimulatory double signals from CD28 and TCR were required for optimal expression of receptor activator of nuclear factor- $\mathrm{kB}$ ligand (RANKL) in periodontal tissues [43]. In the present study, the results showed that PTGS2 was involved in module 3 and could be regulated by FOSB in the regulatory network. Thus, we suggested that PTGS2 might play a critical role in periodontitis progression involving in TCR signaling pathway via interacting with FOSB.

\section{Conclusion}

In conclusion, this study identified several genes (CTSS, PLEK, IRF-8, PTGS2 and FOSB) that involved in the development and progression of periodontitis. CTSS may play an essential role in bone loss associated with periodontitis by interacting with IRF8. Besides, PLEK may contribute to the periodontitis progression via interacting with IRF-8. In addition, PTGS2 may play a critical role in periodontitis progression involving in TCR signaling pathway via interacting with FOSB. Our study may provide theoretical basis for the future investigations of periodontitis. However, further experimental studies are still needed to confirm our results.

\section{Additional file}

Additional file 1: Table S1. All the differentially expressed genes (DEGs) in periodontitis samples with their corresponding modules (DOCX $102 \mathrm{~kb}$ )

\section{Abbreviations}

APC: Antigen presenting cells; BP: Biological processes, CTSS, Cathepsin S, CC, Cellular component; DEGs: Differentially expressed genes, IRF4, Factor 4, FDR, False discovery rate, GO, Gene Ontology; IL-6: Interleukin-6; IL-8: Interleukin-8, KEGG, Kyoto Encyclopedia of Genes and Genomes; KLF4: Kruppel-like factor 4; MMP: Matrix metalloproteinase, MEIS1, Meis homeobox 1; MF: Molecular functions; MAF: Musculoaponeurotic fibrosarcoma oncogene homolog, PLEK, Pleckstrin; PAMPs: Pathogen associated molecular patterns; PPI: Proteinprotein interaction; STRING: Search Tool for the Retrieval of Interacting Genes/Proteins); Th17: T helper cell 17; TLR: Toll like receptors, TFs, Transcription factors.

\section{Competing interests}

The authors declare that they have no competing interests. 


\section{Authors' contributions}

LS conceived of this study, participated in the design, and he performed the statistical analysis. JY carried out the study, together with ZH, collected important background information, and drafted the manuscript. BX participated in the design and helped to draft the manuscript. All authors read and approved the final manuscript.

\section{Acknowledgement}

This study was supported by Minhang District young physician training plan.

\section{Author details}

${ }^{1}$ Department of Stomatology, The Fifth People's Hospital of Shanghai, Fudan University, No.128, Ruili Rd, Minhang District, Shanghai 200240, China. ${ }^{2}$ Department of Endodontics, Shanghai Oral Disease Prevention and Cure Center, Shanghai 200031, China. ${ }^{3}$ Department of Stomatology, The Second Xiangya Hospital of Central South University, Changsha 410011Hunan Province, China.

\section{Received: 2 June 2015 Accepted: 18 August 2015}

\section{Published online: 04 September 2015}

\section{References}

1. Hajishengallis G. Immunomicrobial pathogenesis of periodontitis: keystones, pathobionts, and host response. Trends in immunology. 2014;35:3-11.

2. Genco RJ, Van Dyke TE. Prevention: reducing the risk of CVD in patients with periodontitis. Nature Reviews Cardiology. 2010;7:479-80.

3. Lalla E, Papapanou PN. Diabetes mellitus and periodontitis: a tale of two common interrelated diseases. Nature Reviews Endocrinology. 2011:7:738-48.

4. Madianos PN, Bobetsis YA, Offenbacher S. Adverse pregnancy outcomes (APOs) and periodontal disease: pathogenic mechanisms. Journal of clinical periodontology. 2013;40:S170-80.

5. Demmer RT, Papapanou PN. Epidemiologic patterns of chronic and aggressive periodontitis. Periodontology 2000. 2010;53:28-44.

6. Darveau RP. Periodontitis: a polymicrobial disruption of host homeostasis. Nature Reviews Microbiology. 2010:8:481-90.

7. Cochran DL. Inflammation and bone loss in periodontal disease. Journal of periodontology. 2008;79:1569-76.

8. Graves D, Cochran D. The contribution of interleukin-1 and tumor necrosis factor to periodontal tissue destruction. Journal of periodontology. 2003;74:391-401.

9. Andia DC, de Oliveira NF, Letra AM, Nociti Jr FH, Line SR, de Souza AP. Interleukin-8 gene promoter polymorphism (rs4073) may contribute to chronic periodontitis. Journal of periodontology. 2011;82:893-9.

10. Stoecklin-Wasmer C, Guarnieri P, Celenti R, Demmer R, Kebschull M, Papapanou P. MicroRNAs and their target genes in gingival tissues. Journal of dental research. 2012:91:934-40.

11. Papapanou PN, Behle JH, Kebschull M, Celenti R, Wolf DL, Handfield M, et al. Subgingival bacterial colonization profiles correlate with gingival tissue gene expression. BMC microbiology. 2009;9:221.

12. Kebschull M, Guarnieri P, Demmer R, Boulesteix A-L, Pavlidis P, Papapanou $P$. Molecular differences between chronic and aggressive periodontitis. Journal of dental research. 2013;92:1081-8.

13. Gautier L, Cope L, Bolstad BM, Irizarry RA. affy-analysis of Affymetrix GeneChip data at the probe level. Bioinformatics. 2004;20:307-15.

14. Alttoa A, Kõiv K, Hinsley TA, Brass A, Harro J. Differential gene expression in a rat model of depression based on persistent differences in exploratory activity. European Neuropsychopharmacology. 2010;20:288-300.

15. Smyth GK. Limma: linear models for microarray data, in Bioinformatics and computational biology solutions using $\mathrm{R}$ and Bioconductor. New York: Springer; 2005. p. 397-420.

16. Reiner-Benaim A. FDR control by the $\mathrm{BH}$ procedure for two-sided correlated tests with implications to gene expression data analysis. Biometrical Journal. 2007;49:107-26.

17. Simonoff JS, Hochberg $Y$, Reiser B. Alternative estimation procedures for $\mathrm{Pr}$ $(X<Y)$ in categorized data. Biometrics. 1986;42:895-907.

18. Fury W, Batliwalla F, Gregersen PK, Li W. Overlapping probabilities of top ranking gene lists, hypergeometric distribution, and stringency of gene selection criterion. In: Engineering in Medicine and Biology Society, 2006. EMBS'06. 28th Annual International Conference of the IEEE. 2006. IEEE.
19. Altermann E, Klaenhammer TR. Pathway Voyager: pathway mapping using the Kyoto Encyclopedia of Genes and Genomes (KEGG) database. BMC genomics. 2005;6:60.

20. Franceschini A, Szklarczyk D, Frankild S, Kuhn M, Simonovic M, Roth A, et al. STRING v9. 1: protein-protein interaction networks, with increased coverage and integration. Nucleic acids research. 2013;41:D808-15.

21. Spinelli L, Gambette P, Chapple CE, Robisson B, Baudot A, Garreta H, et al. Clust\&See: a Cytoscape plugin for the identification, visualization and manipulation of network clusters. BioSystems. 2013;113:91-5.

22. Bader GD, Hogue CW. An automated method for finding molecular complexes in large protein interaction networks. BMC Bioinformatics. 2003;4:2.

23. Matys V, Fricke E, Geffers R, Gößling E, Haubrock M, Hehl R, et al. TRANSFAC ${ }^{\oplus}$ : transcriptional regulation, from patterns to profiles. Nucleic acids research. 2003;31:374-8.

24. Moutsopoulos NM, Kling HM, Angelov N, Jin W, Palmer RJ, Nares S, et al. Porphyromonas gingivalis promotes Th17 inducing pathways in chronic periodontitis. Journal of autoimmunity. 2012;39:294-303.

25. Nares S, Moutsopoulos NM, Angelov N, Rangel ZG, Munson PJ, Sinha N, et al. Rapid myeloid cell transcriptional and proteomic responses to periodontopathogenic Porphyromonas gingivalis. The American journal of pathology. 2009;174:1400-14.

26. Zeytun A, Chaudhary A, Pardington P, Cary RB, Gupta G. Induction of cytokines and chemokines by toll-like receptor signaling: strategies for control of infammation. Critical Reviews ${ }^{\mathrm{TM}}$ in Immunology. 2010;30:53-67.

27. Rupanagudi KV, Kulkarni OP, Lichtnekert J, Darisipudi MN, Mulay SR, Schott B, et al. Cathepsin S inhibition suppresses systemic lupus erythematosus and lupus nephritis because cathepsin $S$ is essential for $\mathrm{MHC}$ class II-mediated CD4 T cell and B cell priming. Annals of the rheumatic diseases. 2015;74:452-63.

28. Rauner M, Föger-Samwald U, Kurz M, Brünner-Kubath C, Schamall D, Kapfenberger A, et al. Cathepsin S controls adipocytic and osteoblastic differentiation, bone turnover, and bone microarchitecture. Bone. 2014;64:281-7.

29. Mogi M, Otogoto J. Expression of cathepsin-K in gingival crevicular fluid of patients with periodontitis. Archives of oral biology. 2007:52:894-8.

30. Zhao B, Takami M, Yamada A, Wang X, Koga T, Hu X, et al. Interferon regulatory factor- 8 regulates bone metabolism by suppressing osteoclastogenesis. Nature medicine. 2009;15:1066-71.

31. Buduneli N, Kinane DF. Host-derived diagnostic markers related to soft tissue destruction and bone degradation in periodontitis. Journal of clinical periodontology. 2011;38:85-105.

32. Wang Y, Baron RM, Zhu G, Joo M, Christman JW, Silverman ES, et al. PU. 1 regulates cathepsin $S$ expression in professional APCs. The Journal of Immunology. 2006;176:275-83.

33. Jackson S, Sugiman-Marangos S, Cheung K, Junop M. Crystallization and preliminary diffraction analysis of truncated human pleckstrin. Acta Crystallographica Section F: Structural Biology and Crystallization Communications. 2011;67:412-6.

34. Ding Y, Kantarci A, Badwey JA, Hasturk H, Malabanan A, Van Dyke TE. Phosphorylation of pleckstrin increases proinflammatory cytokine secretion by mononuclear phagocytes in diabetes mellitus. The Journal of Immunology. 2007;179:647-54.

35. Ueki K, Tabeta K, Yoshie H, Yamazaki K. Self-heat shock protein 60 induces tumour necrosis factor-a in monocyte-derived macrophage: possible role in chronic inflammatory periodontal disease. Clinical \& Experimental Immunology. 2002;127:72-7.

36. Qiao Y, Giannopoulou EG, Chan CH, Park S-h, Gong S, Chen J, et al. Synergistic activation of inflammatory cytokine genes by interferon- $\gamma$ induced chromatin remodeling and Toll-like receptor signaling. Immunity. 2013;39:454-69.

37. Bar-Or A, Fawaz L, Fan B, Darlington PJ, Rieger A, Ghorayeb C, et al. Abnormal B-cell cytokine responses a trigger of T-cell-mediated disease in MS? Annals of neurology. 2010;67:452-61.

38. Walker MR, Brown SL, Riehl TE, Stenson WF, Stappenbeck TS. Growth factor regulation of prostaglandin-endoperoxide synthase 2 (Ptgs2) expression in colonic mesenchymal stem cells. Journal of Biological Chemistry. 2010;285:5026-39.

39. Zhang S, Barros S, Niculescu M, Moretti A, Preisser J, Offenbacher S. Alteration of PTGS2 promoter methylation in chronic periodontitis. Journal of dental research. 2010;89:133-7. 
40. Grueter BA, Robison AJ, Neve RL, Nestler EJ, Malenka RC. $\triangle$ FosB differentially modulates nucleus accumbens direct and indirect pathway function. Proceedings of the national academy of sciences. 2013;1 10:1923-8.

41. Brockmeyer C, Paster W, Pepper D, Tan CP, Trudgian DC, McGowan S, et al. T cell receptor (TCR)-induced tyrosine phosphorylation dynamics identifies THEMIS as a new TCR signalosome component. Journal of Biological Chemistry. 2011;286:7535-47.

42. Sreeramkumar V, Fresno M, Cuesta N. Prostaglandin E2 and T cells: friends or foes\&quest. Immunology and cell biology. 2012;90:579-86.

43. Chen B, Wu W, Sun W, Zhang Q, Yan F, Xiao Y. RANKL expression in periodontal disease: where does RANKL come from? BioMed research international. 2014;2014:731039.

\section{Submit your next manuscript to BioMed Central and take full advantage of:}

- Convenient online submission

- Thorough peer review

- No space constraints or color figure charges

- Immediate publication on acceptance

- Inclusion in PubMed, CAS, Scopus and Google Scholar

- Research which is freely available for redistribution 